\title{
Sunlamp Exposure
}

National Cancer Institute

\section{Source}

National Cancer Institute. Sunlamp Exposure. NCI Thesaurus. Code C44450.

Using an artificial light source, either sunlamp or sunbed, to generate a tanned skin. 